\title{
Compensation of carrier lifetime in double-pass semiconductor optical amplifiers
}

\begin{abstract}
Bi-directional light propagation is expected to enable enhanced functionality of all-optical signal processing operations compared to unidirectional approaches. In this work, we report on compensation of slow gain recovery in semiconductor optical amplifiers (SOAs) in a double-pass condition. The unsaturated gain of the employed SOA is increased by $12.6 \mathrm{~dB}$, and the corresponding normalized conjugate output of four-wave mixing is enhanced by 16.3 $\mathrm{dB}$. The theory attributes this efficiency improvement to the unsaturated gain enlargement which, in turn, compensates for the inherently long carrier lifetimes of SOAs by $50 \%$. The saturation output power remains virtually unchanged.
\end{abstract}

Keyword: Carrier lifetime; Double-pass; Four-wave mixing; Semiconductor optical amplifier; Unsaturated gain 